УДК 621.3.01

\title{
О ВЗАИМНОМ ВЛИЯНИИ ЭЛЕКТРОМАГНИТНЫХ ВЕКТОРОВ ДВИЖУЩИХСЯ ЭЛЕКТРИЧЕСКИХ СИСТЕМ
}

\author{
Канд. техн. наук П.Я. Придубков
}

\section{ПРО ВЗАСМНИЙ ВПЛИВ ЕЛЕКТРОМАГНІТНИХ ВЕКТОРІВ РУХОМИХ ЕЛЕКТРИЧНИХ СИСТЕМ}

\author{
Канд. техн. наук П.Я. Придубков \\ ON THE MUTUAL INFLUENCE OF ELECTROMAGNETIC VECTORS MOVING \\ ELECTRICAL SYSTEMS
}

\author{
Cand. of techn. sciences P. Pridubkov
}

Показана взаимосвязь электромагнитных параметров различных инерциальных электротехнических систем с помощью инвариантных операций четырёхмерного обобщения, установлень дифференциальные аналитические зависимости, соответствующие уравнениям закона Ома электрических иепей, позволяющие описать взаимосвязь и взаимное влияние друг на друга электрических и магнитных векторов движушихся электрических систем.

Ключевые слова: четырёхмерное векторное исчисление, инвариантные операции четырёхмерного обобщения операторов Лапласа и четырёхмерной дивергенции.

Показано взаємозв'язок електромагнітних параметрів різних інериіальних електротехнічних систем за допомогою інваріантних операцій чотиривимірного узагальнення, встановлено диференщіальні аналітичні залежності, відповідні рівнянням закону Ома електричних кіл, що дають змогу описати взаємозв'язок $і$ взаємний вплив один на одного електричних і магнітних векторів електричних систем, щуо рухаються.

Ключові слова: чотиривимірне векторне числення, інваріантні операції чотиривимірного узагальнення операторів Лапласа і чотиривимірної дивергенції.

Intercommunication of electromagnetic parameters of different inertia electrical engineering systems by invariant operations of 4-measured generalization is shown, differential analytical dependences proper to equalizations of law of Ohm of electric chains are set, allowing to describe intercommunication and mutual influencing on each other of vectors of the locomotive electric systems electric and magnetic.

Keywords: four-dimensional vector calculus operations four-invariant generalization of Laplace and four-divergence.

Вступление. Использование четырёхмерного пространства при рассмотрении электромагнитных процессов электротехнических устройств железнодорожного 
транспорта, позволяющее повысить эффективность их функционирования, предполагает необходимость исследования дифференциальных зависимостей, описывающих основные законы и уравнения электротехники основными векторами электродинамики [1].

Электрическая цепь представляет собой совокупность проводников, приёмников и источников электрической энергии, соединённых между собой в соответствии с заданной схемой и образующих пути для протекания электрического тока. В электротехнике уделяется большое внимание движущимся электрическим цепям, к которым относятся электрические системы железнодорожного транспорта.

Выведенные для неподвижных электрических цепей соотношения, описывающие электромагнитные процессы, при переходе от одной неподвижной инерциальной системы отсчета (ИСО) к другой ИСО, движущейся относительно первой, преобразуются в соответствии с основными положениями релятивистской электродинамики.

Использование электродинамических потенциалов, объединённых в одно четырёхмерное образование (четырёхвектор), и введение четырёхвектора плотности тока позволяют свести уравнения Максвелла к уравнению для четырёхвектора потенциала с инвариантными операциями четырёхмерного обобщения оператора Лапласа и оператора четырёхмерной дивергенции [2]. Изотропия четырёхмерного пространства имеет адекватное выражение в четырёхмерном векторном исчислении, согласно которому уравнения Максвелла являются инвариантными, то есть выглядят одинаково во всех инерциальных системах отсчёта.

Четырёхмерная запись уравнений Максвелла позволяет выявить взаимосвязь электромагнитных электротехнических систем различных
ИСО. Кроме того, данная запись упрощает процесс рассмотрения дифференциальных аналитических (функциональных) зависимостей, соответствующих обмену сигналами (информацией) между движущимися относительно друг друга объектами.

Стало быть, исследование и уточнение дифференциальных зависимостей основных параметров движущихся электрических цепей, их четырёхмерных интерпретаций является актуальной проблемой, позволяющей повысить эффективность функционирования и точность расчёта электротехнических устройств железнодорожного транспорта.

Основная часть. Электромагнитное поле - это совокупность изменяющихся во времени, взаимно связанных, обуславливающих друг друга электрического и магнитного полей. Электромагнитные процессы, протекающие в данном поле, описываются системой уравнений Максвелла [3].

Уравнение, выражающее связь между ротором напряжённости магнитного поля и плотностью тока в той же точке поля, является первым уравнением Максвелла, которое представляет собой дифференциальную форму уравнения закона полного тока:

$$
\operatorname{rotH}=\delta
$$

здесь $\boldsymbol{\delta}$ - вектор плотности полного тока, составляющими которого являются вектор плотности тока проводимости $\delta_{\text {пр }}=\mathrm{\gamma E}$ и вектор плотности тока смещения $\boldsymbol{\delta}_{\mathrm{cm}}=\frac{\partial \mathbf{D}}{\partial \mathrm{t}}$ :

$$
\delta=\delta_{\text {пр }}+\delta_{\text {см }}
$$

Третьим уравнением Максвелла является дифференциальная форма уравнения теоремы Гаусса: 


$$
\nabla D=\rho .
$$

Вышеуказанные уравнения представляют собой первую группу уравнений Максвелла, описывающих процессы электромагнитного поля:

$$
\nabla \mathrm{D}=\rho, \quad \operatorname{rot} \mathrm{H}=\boldsymbol{\delta} .
$$

Координаты $\mathrm{x}, \mathrm{y}, \mathrm{z}$ и $\mathrm{t}$ в уравнениях (1) можно заменить мировыми координатами $\mathrm{x}_{1}=\mathrm{x}, \quad \mathrm{x}_{2}=\mathrm{y}, \quad \mathrm{x}_{3}=\mathrm{z} \quad$ и $\mathrm{x}_{4}=\mathrm{t}$ соответственно, с помощью которых все события в мире определяются в пространстве и времени, кроме того, необходимо принять во внимание четырёхмерный характер вектора [2] плотности тока, компоненты которого равны

$$
\delta_{\mu}=(\delta, \mathrm{jc} \rho),
$$

при этом $\mathrm{X}$ - составляющая первого уравнения Максвелла запишется в виде

$$
\frac{\partial \mathrm{H}_{\mathrm{z}}}{\partial x_{\mathrm{y}}}-\frac{\partial \mathrm{H}_{\mathrm{y}}}{\partial x_{\mathrm{z}}}=\frac{\partial \mathrm{D}_{\mathrm{x}}}{\partial \mathrm{t}}+\delta_{\mathrm{x}}
$$

Если учитывать, что векторы H и D образуют свой особый четырёхмерный тензор $f=(H,-c D)$, то уравнение (2) имеет следующий четырёхмерный вид:

$$
\frac{\partial \mathrm{f}_{12}}{\partial \mathrm{x}_{2}}+\frac{\partial \mathrm{f}_{13}}{\partial \mathrm{x}_{3}}+\frac{\partial \mathrm{f}_{14}}{\partial \mathrm{x}_{4}}=\delta_{1}
$$

Таким же образом могут быть преобразованы и две другие составляющие первого уравнения Максвелла. Данное преобразование в общем виде для $\mu=1,2,3$ записывается следующим образом:

$$
\sum_{\mathrm{v}=1}^{4} \frac{\partial \mathrm{f}_{\mu \mathrm{v}}}{\partial \mathrm{x}_{\mathrm{v}}}=\delta_{\mu}
$$

При распространении уравнения (3) и на значение $\mu=4$ в соответствии с компонентами четвёртой строки тензора $\mathbf{f}=(\mathbf{H},-\mathbf{C D})$ третьему уравнению Максвелла соответствует выражение

$$
\mathrm{jc}\left(\frac{\partial \mathrm{D}_{\mathrm{x}}}{\partial \mathrm{x}}+\frac{\partial \mathrm{D}_{\mathrm{y}}}{\partial \mathrm{y}}+\frac{\partial \mathrm{D}_{\mathrm{z}}}{\partial \mathrm{z}}\right)=\delta_{4} .
$$

Операция $\sum \frac{\partial}{\partial \mathrm{x}_{\mathrm{v}}}$, введённая в выражении (3), в общем тензорном анализе называется «дифференцированием с последующим свертыванием». Она превращает четырёхтензор в четырёхвектор и обозначается Div:

$$
\operatorname{Div}_{\mu} f=\sum_{v=1}^{4} \frac{\partial f_{\mu v}}{\partial x_{v}}=\delta_{\mu}
$$

Для каждого антисимметричного четырёхтензора $\mathrm{T}_{\mathrm{v \mu}}$ выполняется общее соотношение:

$$
\operatorname{DivDivT}_{\mathrm{v} \mu}=0
$$

где $\operatorname{Div}=\nabla_{\mathrm{v}}=\sum_{\mathrm{v}}^{4} \frac{\partial}{\partial \mathbf{x}_{\mathrm{v}}} \quad$ - четырёхмерная дивергенция.

Из последнего выражения следует, что дивергенция четырёхвектора плотности тока должна обращаться в нуль.

Компоненты трёхмерных векторов $\mathrm{H}$ и D преобразуются по правилу преобразования компонент четырёхтензора $\mathrm{f}=(\mathrm{H},-\mathrm{CD})$, поэтому при переходе от одной ИСО к другой все компоненты векторов электрического поля меняют свою величину и направление под действием компонент векторов магнитного поля и наоборот. Неизменными остаются только «продольные компоненты», то есть 
компоненты по направлению относительного движения.

Ток электрического смещения возникает в любой диэлектрической (вещественной) среде распространения электромагнитного поля, в том числе и в вакууме, при изменении напряжённости электрического поля во времени:

$$
\delta_{\mathrm{CM}}=\frac{\partial \mathrm{D}}{\partial \mathrm{t}}=\frac{\partial\left(\varepsilon_{\alpha} \mathrm{E}\right)}{\partial \mathrm{t}} .
$$

Током проводимости является упорядоченное движение свободных электрических зарядов в проводящей среде. Данный ток является скаляром алгебраического характера:

$$
\mathrm{i}=\int_{\mathrm{S}} \delta_{\Pi \mathrm{p}} \mathrm{d} \mathbf{S}
$$

где $\delta_{\text {пр }}$ - поверхностная плотность тока проводимости;

dS - элемент площадки поперечного сечения.

В соответствии с дифференциальной формой закона Ома плотность тока проводимости определяется соотношением

$$
\delta_{\text {пр }}=\gamma E \text {. }
$$

Поэтому первому уравнению Максвелла соответствует выражение

$$
\operatorname{rot} \mathbf{H}=\boldsymbol{\delta}_{\text {пр }}+\frac{\partial \mathbf{D}}{\partial \mathrm{t}}
$$

или

$$
\operatorname{rotH}=\mathrm{VE}+\frac{\partial \mathbf{D}}{\partial \mathrm{t}}
$$

\footnotetext{
Согласно одному из основных положений векторного анализа дивергенция ротора равна нулю (divrotH =0), стало быть:
}

$$
\nabla \boldsymbol{\delta}_{\text {пр }}+\nabla \frac{\partial \mathbf{D}}{\partial \mathrm{t}}=0
$$

Если учесть независимость дифференцирования по времени от пространственного дифференцирования, то

$$
\nabla \boldsymbol{\delta}_{\text {пр }}+\frac{\partial}{\partial \mathrm{t}}(\nabla \mathbf{D})=0 .
$$

Так как в соответствии с дифференциальной формой уравнения теоремы Гаусса $\nabla \mathbf{D}=\rho$, поэтому

$$
\nabla \delta_{\text {пр }}+\frac{\partial \rho}{\partial t}=0
$$

Последнее выражение является четырёхмерной дивергенцией четырёхвектора плотности тока, которая, как следует из выражений (4) и (5), равна нулю [4]:

$$
\nabla_{\mu} \delta_{\mu}=\left(\nabla \delta_{\text {пр }}+\nabla_{\mathrm{T}} \delta_{\mathrm{T}}\right),
$$

здесь $\quad \nabla_{\mathrm{T}}=\frac{\partial}{\partial \mathrm{x}_{4}}=\frac{\partial}{\mathrm{jc} \partial \mathrm{t}} \quad$ - временная компонента четырёхмерной дивергенции;

$\delta_{\mathrm{T}}=\mathrm{jc \rho}-$ временная компонента четырёхвектора плотности тока проводимости.

Из первого уравнения Максвелла следует, что магнитное поле может возникать не только в результате наличия тока проводимости, но также в результате изменения электрического поля во времени. Основное различие между этими токами усматривается в том, что первый возникает в результате движения электрических зарядов, второй же - в результате изменения электрического поля во времени, то есть в отличии природы их возникновения. 
Между тем и ток проводимости, и ток смещения можно трактовать как изменение во времени потока вектора электрического смещения.

При протекании в проводящих средах постоянного тока одни электрические заряды сменяются другими, такими же, как и в предыдущие моменты времени. Картина стационарного электрического поля постоянного тока в макроскопическом смысле повторяется в смежные моменты времени. Стало быть, поле, созданное в проводящих средах разделяющимися зарядами, носит как бы статический характер [3] и в любой из смежных моментов времени его можно рассматривать как электростатическое поле, а среды - диэлектрическими.

Следовательно, можно предположить, что одна из двух сред стационарного электрического поля постоянного тока является проводящей, а другая диэлектрической. На границе раздела данных сред в рассматриваемый момент времени должно выполняться условие

$$
\sigma_{\mathrm{s}}=\mathrm{D}_{\mathrm{n}}
$$

здесь $\sigma_{\mathrm{s}}=\frac{\mathrm{dq}_{\mathrm{s}}}{\mathrm{dS}}-$ поверхностная плотность свободного заряда в данный момент времени на поверхности одной из проводящих сред;

$D_{n}$ - модуль вектора электрического смещения другой проводящей среды, рассматриваемой в качестве диэлектрической.

Поток вектора $\mathbf{D}_{\text {п }}$ электрического смещения сквозь любую поверхность $S$ определяется интегралом [5]

$$
\Psi_{\text {эл.п }}=\int_{S} D_{n} \mathrm{~d} S \text {. }
$$

Если поверхность (S) является замкнутой, то в соответствии с теоремой Гаусса «поток вектора электрического смещения через любую замкнутую поверхность, окружающую некоторый объём, равен алгебраической сумме свободных зарядов $\Sigma q_{s}$, находящихся внутри этой поверхности» [2]:

$$
\Psi_{\text {эл }}=\oint_{\mathrm{S}} \mathrm{D}_{\mathrm{n}} \mathrm{d} S=\Sigma \mathrm{q}_{\mathrm{s}} .
$$

Разрабатывая теорию электромагнитного поля, Максвелл предположил, что теорема Гаусса остаётся справедливой и для любого электрического поля, связанного или несвязанного с неподвижными или как угодно движущимися зарядами (постулат Максвелла). Все выводы, вытекающие из данного допущения, не противоречат известным современной науке экспериментальным фактам. Это даёт основание утверждать, что в пределах современных знаний теорема Гаусса справедлива для любого электрического поля в любых условиях, в том числе и для стационарного электрического поля постоянного тока в проводящей среде.

Стало быть, заряды, входящие в правую часть соотношения (6), могут быть как статическими, так и меняющимися во времени. Последние обуславливают изменение во времени потока вектора электрического смещения. Поэтому при рассмотрении стационарного электрического поля постоянного тока в проводящей среде, как электростатического, можно констатировать, что

$$
\frac{\partial \Psi_{\text {эл.п }}}{\partial \mathrm{t}}=\frac{\partial}{\partial \mathrm{t}} \oint_{\mathrm{S}} \mathbf{D}_{\Pi} \mathrm{d} \mathbf{S}=\frac{\partial\left(\Sigma \mathrm{q}_{\mathrm{S}}\right)}{\partial \mathrm{t}} .
$$

Учитывая, что пространственные координаты от времени не зависят, в правой части последнего выражения можно изменить порядок интегрирования и дифференцирования, поэтому 


$$
\frac{\partial \Psi_{\text {эл.п }}}{\partial \mathrm{t}}=\oint_{\mathrm{S}} \frac{\partial \mathbf{D}_{\text {п }}}{\partial \mathrm{t}} \mathrm{d} \mathbf{S}=\frac{\partial\left(\sum \mathrm{q}_{\mathrm{s}}\right)}{\partial \mathrm{t}} .
$$

Изменение во времени (упорядоченное движение) свободных электрических зарядов является током проводимости $\mathrm{i}_{\text {пр }}$ :

$$
\frac{\partial\left(\Sigma \mathrm{q}_{\mathrm{s}}\right)}{\partial \mathrm{t}}=\mathrm{i}_{\text {пр }},
$$

стало быть:

$$
\frac{\partial \Psi_{\text {эл.п }}}{\partial \mathrm{t}}=\frac{\partial\left(\Sigma \mathrm{q}_{\mathrm{s}}\right)}{\partial \mathrm{t}}=\mathrm{i}_{\text {пр }} .
$$

Следовательно, изменение во времени потока вектора $\mathbf{D}_{n}$ электрического смещения $\Psi_{\text {эл.п }}$ в проводящей среде $\left(\frac{\partial \Psi_{\text {эл.п }}}{\partial \mathrm{t}}\right)$ можно трактовать как ток проводимости:

$$
\frac{\partial \Psi_{\text {эл.п }}}{\partial \mathrm{t}}=\mathrm{i}_{\text {пр }} .
$$

Электрический ток проводимости является потоком вектора плотности тока [3]:

$$
\mathrm{i}_{\text {пр }}=\oint_{\mathrm{S}} \boldsymbol{\delta}_{\text {пр }} \mathrm{d} \mathbf{S}
$$

Данный поверхностный интеграл в соответствии с теоремой Остроградского может быть преобразован в объёмный:

$$
\oint_{S} \delta_{п р} d S=\int_{V} \operatorname{div} \delta_{п р} d V
$$

Исток вектора электрического смещения $\mathbf{D}_{\text {п }}$ через замкнутую поверхность проводящей среды (исток потока этого вектора) определяется теоремой Гаусса в дифференциальной форме. Если электрическое поле создаётся статическими зарядами, то

$$
\operatorname{div}_{n}=\rho
$$

Стало быть, истоку электрического поля меняющихся во времени зарядов соответствует выражение

$$
\frac{\partial\left(\operatorname{div} D_{n}\right)}{\partial t}=\frac{\partial \rho}{\partial t} .
$$

Независимость пространственных координат от времени позволяет изменить порядок дифференцирования:

$$
\operatorname{div} \frac{\partial \mathbf{D}_{n}}{\partial \mathrm{t}}=\frac{\partial \rho}{\partial \mathrm{t}}
$$

Для произвольно выбранного объёма V проводящей среды можно констатировать, что

$$
\int_{\mathrm{V}} \operatorname{div} \frac{\partial \mathbf{D}_{\Pi}}{\partial \mathrm{t}} \mathrm{dV}=\frac{\partial}{\partial \mathrm{t}} \int_{\mathrm{V}} \rho \mathrm{dV}
$$

или

$$
\int_{V} \operatorname{div} \frac{\partial D_{n}}{\partial t} d V=\frac{\partial\left(\Sigma q_{s}\right)}{\partial t}
$$

Если учесть уравнение (9), а также и то, что

$$
\frac{\partial\left(\Sigma q_{s}\right)}{\partial t}=i_{п р}=\oint_{S} \delta_{\text {пр }} d S
$$

то соотношение (10) следует записать как

$$
\int_{V} \operatorname{div} \frac{\partial \mathbf{D}_{\Pi}}{\partial \mathrm{t}} \mathrm{dV}=\int_{\mathrm{V}} \operatorname{div} \delta_{\Pi p} \mathrm{dV}
$$

Так как объём V был выбран произвольно, то последнее равенство (11) не зависит от пределов интегрирования. 
Поэтому его подынтегральные функции равны между собой:

$$
\operatorname{div} \frac{\partial \mathbf{D}_{\Pi}}{\partial \mathrm{t}}=\operatorname{div}_{\text {пр }}
$$

тогда

$$
\frac{\partial \mathbf{D}_{п}}{\partial \mathrm{t}}=\boldsymbol{\delta}_{\text {пр }} .
$$

Левая часть последнего уравнения (12) представляет собой вектор плотности тока смещения $\left(\frac{\partial \mathbf{D}_{n}}{\partial \mathrm{t}}=\boldsymbol{\delta}_{\mathrm{cm}}\right)$, стало быть: $\boldsymbol{\delta}_{\text {пр }}=\boldsymbol{\delta}_{\mathrm{cm}}$, что полностью соответствует уравнению непрерывности полного тока, согласно которому полный ток $\delta_{n}$ всегда замкнут:

$$
\oint_{S} \delta_{n} d S=0
$$

Для однородных и изотропных проводящих сред векторы электрической индукции и напряжённости должны быть связаны соотношением

$$
D_{n}=\varepsilon_{a_{n}} E_{n},
$$

здесь $\varepsilon_{\mathrm{a}_{n}}$ - диэлектрическая проницаемость проводящей среды.

Поэтому вектор плотности смещения может быть определён выражением

$$
\delta_{\mathrm{cm}}=\varepsilon_{\mathrm{a}_{\mathrm{n}}} \frac{\partial \mathbf{E}_{\Pi}}{\partial \mathrm{t}},
$$

которое справедливо для любого объёма

$$
\int_{V} \delta_{c M} d V=\int_{V} \varepsilon_{a_{n}} \frac{\partial E_{n}}{\partial t} d V
$$

Так как

$$
\int_{V} d V=\iint_{S l} d S d l
$$

следовательно

$$
\int_{S} \delta_{c M} d S=\varepsilon_{a_{n}} \frac{\int_{S} d S}{\int_{l} d l} \frac{\partial}{\partial t} \int_{l} E_{n} d l .
$$

Если учесть, что $\int_{S} \boldsymbol{\delta}_{\text {см }} \mathrm{d} \mathbf{S}=\mathrm{i}_{\mathrm{c}}$, как и то, что $\varepsilon_{\mathrm{a}_{\text {n }}}$ является удельной ёмкостью, а значит, $\quad \varepsilon_{a_{n}} \frac{\int_{\mathrm{S}} \mathrm{d} S}{\int_{l} \mathrm{dl}}=\mathrm{C}, \quad$ a также, что $\int_{\mathbf{l}} \mathrm{E}_{\mathrm{n}} \mathrm{dl}=\mathrm{u}_{\mathrm{c}}$, то можно констатировать:

$$
\mathrm{i}_{\mathrm{c}}=\mathrm{C} \frac{\partial \mathrm{u}_{\mathrm{c}}}{\partial \mathrm{t}} \text {. }
$$

Уравнение (14) может быть описано при помощи комплексных величин следующим выражением:

$$
\dot{\mathrm{i}}_{\mathrm{c}}=\mathrm{j} \omega \mathrm{CU},
$$

которое является формулой, соответствующей закону Ома для диэлектрического участка среды распространения электромагнитного поля.

$$
\text { Уравнение }
$$

дифференциальной

является вышеуказанного закона, в котором плотность тока смещения выражена через вектор напряжённости электрического поля. Стало быть, данное уравнение позволяет учитывать влияние электромагнитных параметров одной ИСО на электромагнитные параметры другой ИСО с помощью силы Лоренца или её магнитного аналога, используя тензор 
четырёхмерного

$F_{\mu v}=\left(B,-\frac{j}{c} E\right)$.

Уравнение (12) для однородных и изотропных сред распространения электрического поля может быть записано следующим образом:

$$
\frac{\partial\left(\varepsilon_{a_{n}} E_{n}\right)}{\partial t}=\delta_{n р} .
$$

Вектор плотности тока $\boldsymbol{\delta}_{\text {пр }}$ электрического поля в проводящей среде определяется на основании закона Ома в дифференциальной форме [3] выражением

$$
\delta_{n p}=\gamma E_{n},
$$

Напряжённость $\mathbf{E}_{n}$ электрического поля постоянного тока в проводящей среде является величиной постоянной $\left(\mathbf{E}_{n}=\right.$ const. $)$, поэтому

$$
\frac{\partial\left(\varepsilon_{a_{n}} E_{n}\right)}{\partial t}=\frac{\partial \varepsilon_{a_{n}}}{\partial t} \mathbf{E}_{n} .
$$

Таким образом:

$$
\frac{\partial \varepsilon_{a_{n}}}{\partial t} E_{n}=\gamma E_{n}
$$

Следовательно, параметры проводящей среды, её диэлектрическая проницаемость $\quad \varepsilon_{a_{n}}$ и удельная проводимость $ү$ связаны соотношением

$$
\frac{\partial \varepsilon_{\mathrm{a}_{\mathrm{n}}}}{\partial \mathrm{t}}=\mathrm{\gamma} \text {. }
$$

a дифференциальной форме закона Ома соответствует выражение

$$
\delta_{\mathrm{cm}}=\frac{\partial \varepsilon_{\mathrm{a}_{\mathrm{n}}}}{\partial \mathrm{t}} \mathbf{E}_{\mathrm{n}}
$$

Выводы. Таким образом, полученные аналитические зависимости (13) и (15), описывающие дифференциальные уравнения закона Ома, обеспечивают повышение эффективности функционирования электротехнических систем железнодорожного транспорта. Это обусловлено тем, что при переходе от электромагнитных параметров одной ИСО к аналогичным параметрам другой движущейся ИСО, данные выражения позволяют учитывать влияние электрических параметров первой ИСО на магнитные параметры второй ИСО и наоборот.

\section{Список использованных источников}

1. Придубков, П.Я. Электромагнитные процессы железнодорожных систем и четырёхмерное пространство [Текст] / П.Я. Придубков // Залізничний транспорт України. 2012. - № 5. - С. 35-39

2. Зоммерфельд, А. Электродинамика [Текст] / А. Зоммерфельд.- М.: Издательство иностранной литературы, 1958. -502 с.

3. Бессонов, Л.А. Теоретические основы электротехники. Электромагнитное поле [Текст] / Л.А. Бессонов. - М.: Высшая школа, 1986. - 263 с.

4. Мейерович, Э.А. Методы релятивисткой электродинамики в электротехнике и электрофизике [Текст] / Э.А. Мейерович, Б.Э. Мейерович. - М.: Энергоатомиздат. 1987. $232 \mathrm{c}$. 
Рухомий склад залізниць

5. Тамм, И.Е. Основы теории электричества [Текст] / И.Е. Тамм. - М.: Издательство физико-математической литературы, 2003. - 616 с.

Придубков Павло Якович, кандидат технічних наук, доцент кафедри електротехніки та електричних машин Української державної академії залізничного транспорту.

Pridubkov P., cand. of techn. sciences, associate professor Ukrainian State Academy of Railway Transport. 